\title{
Cognitive-Behavioral Correlates of Psychological Symptoms Among Relatives of Missing Persons
}

\author{
Lonneke I. M. Lenferink ${ }^{1,2} \cdot$ Jos de Keijser $^{1} \cdot$ Ineke Wessel $^{1} \cdot$ Paul A. Boelen $^{2,3}$
}

Published online: 17 September 2018

(C) The Author(s) 2018

\begin{abstract}
The disappearance of significant others is associated with an increased risk of prolonged grief disorder (PGD), post-traumatic stress disorder (PTSD), and major depressive disorder (MDD). Enhancing knowledge about cognitive-behavioral correlates of PGD, PTSD, and MDD may generate valuable information for developing interventions for relatives of missing persons. We aimed to examine whether prior findings, indicating that cognitive-behavioral variables are related to distress among bereaved individuals and generalize to relatives of missing persons. Relatives of missing persons $(n=134)$ completed self-report measures of negative cognitions, avoidance behaviors, PGD, PTSD, and MDD. Multilevel analysis was used. Cognitive-behavioral variables explained 40 to $60 \%$ of the additional variance in PGD, PTSD, and MDD levels over and above sociodemographic variables. Similar to bereaved individuals, relatives of missing persons who tend to engage in negative cognitions and avoidance behaviors are more likely to experience elevated psychopathology levels. Addressing cognitive-behavioral variables in treatment may be beneficial.
\end{abstract}

Keywords Ambiguous loss $\cdot$ Disappearance $\cdot$ Missing people $\cdot$ Traumatic loss ·

Bereavement

The long-term disappearance of a significant other is a unique type of loss, due to uncertainty about the whereabouts of the missing loved one. Not knowing whether the separation is temporary or permanent may complicate the grieving process (Boss

Lonneke I. M. Lenferink

1.i.m.lenferink@rug.nl

Jos de Keijser

a.de.keijser@rug.nl

Ineke Wessel

j.p.wessel@rug.nl

Paul A. Boelen

p.a.boelen@uu.nl

Extended author information available on the last page of the article 
2006). There is evidence that similar to people confronted with other potential traumatic losses (Kristensen et al. 2012), relatives of missing persons have an increased risk of the development of symptoms of prolonged grief disorder (PGD), ${ }^{1}$ post-traumatic stress disorder (PTSD), and major depressive disorder (MDD) (Heeke and Knaevelsrud 2015). For example, a study among people confronted with the disappearance of a family member or friend due to political repression showed that interview-based rates of PGD (23.3\%), PTSD (67.1\%), and MDD (68.5\%) are comparable to homicidally bereaved individuals (Heeke et al. 2015). PGD symptoms (e.g., intense sorrow and preoccupation with the deceased loved one) differ from normal grief reactions in that they last longer and are more intense (Maciejewski et al. 2016). In addition, the hallmark of PGD (i.e., longing for the deceased) is distinct from key symptoms of PTSD (i.e., intrusion symptoms) and MDD (i.e., sadness), which has been supported by factor analytic research (e.g., Prigerson et al. 1996).

Literature regarding correlates of psychological symptoms, including symptoms of PGD, PTSD, and MDD, in relatives of long-term missing persons is scarce and is primarily focused on sociodemographic correlates (Heeke and Knaevelsrud 2015; Lenferink et al. 2017). Moreover, studies exploring correlates that are amendable to therapeutic change are lacking. Gaining insights into these correlates is relevant for developing psychological treatment for relatives of missing persons in need of professional support.

Several theories emphasize the importance of negative cognitions and avoidance behaviors in the development and persistence of PGD, PTSD, and MDD (e.g., Beck 1987; Ehlers and Clark 2000; Shear and Shair 2005). Multiple studies, based on a cognitive behavioral model of PGD (Boelen et al. 2006), showed that negative cognitions and avoidance behavior are related to symptom levels of not only PGD but also PTSD and MDD concurrently and prospectively in people who experienced the death of a loved one (Boelen et al. 2015; Boelen and Eisma 2015; Boelen and van den Bout 2010; Eisma et al. 2013; van der Houwen et al. 2010). Negative cognitions may include a negative self-view (e.g., "Since (-) is dead, I feel worthless"), negative view on one's own life (e.g., "My life is meaningless since (-) died"), a pessimistic view on the future (e.g., "I don't have confidence in the future"), and catastrophic misinterpretations of one's own grief reactions (e.g., "Once I would start crying, I would lose control"). Avoidance behavior includes both anxious avoidance and depressive avoidance. Anxious avoidance refers to avoidance of loss-related stimuli out of fear that confrontation with these stimuli will be unbearable. Depressive avoidance behavior includes withdrawal from different social/recreational activities fueled by the belief that these activities are pointless and/or unfulfilling (Boelen et al. 2006).

To the best of our knowledge, cognitive-behavioral correlates of psychopathology in relatives of missing persons have not been studied previously. As noted, knowledge about these correlates may inform development and refinement of treatment interventions. Accordingly, the aim of this study - conducted in the Netherlands - was to examine whether prior findings, indicating that cognitive-behavioral variables are related to emotional distress after the death of loved one generalize to relatives of

\footnotetext{
${ }^{1}$ PGD resembles persistent complex bereavement disorder as recently included as condition for further study in the fifth edition of the Diagnostic and Statistical Manual of Mental Disorders (DSM-5, American Psychiatric Association [APA] 2013; see Maciejewski et al. 2016).
} 
long-term missing persons. We expected that negative cognitions (about one's self, life, future, and catastrophic misinterpretations of one's own grief reactions) and anxious and depressive avoidance behaviors would explain amounts of variance in symptom levels of PGD, PTSD, and MDD over and above sociodemographic variables.

\section{Method}

\section{Recruitment}

The present data come from 134 participants in a research project on the correlates and treatment of psychopathology in relatives of missing persons (Lenferink et al. 2017; Lenferink et al. 2017; Lenferink et al. 2018; Lenferink et al. 2016). Inclusion criteria were the following: (a) being a nuclear or extended family member, spouse, or friend of a person who has been missing for at least 3 months, (b) fluent in Dutch language, and (c) being at least 18 years of age. The following definition of a missing person was used: "Anyone whose whereabouts is unknown whatever the circumstances of disappearance. They will be considered missing until located and their well-being or otherwise established" (Association of Chief Police Officers 2010, p. 15). Paper-andpencil questionnaire data were collected between July 2014 and January 2016. Participants were recruited via invitation letters sent by representatives of a Dutch television show about missing persons $(26.9 \%)$ and peer support organizations $(22.4 \%)$, and referral via Victim Support the Netherlands - a non-governmental organization for victim support- $(15.7 \%)$. Others were approached through family members or friends $(26.1 \%)$ or other recruitment procedures (e.g., media-attention) $(9.0 \%)$. These recruitment rates were based on the responses to an item about how the respondents were referred to this study. We cannot rule out that participants could have received multiple invitations to participate from different sources. Approval for conducting the study was obtained from a local ethics review board. All participants gave written informed consent.

\section{Participants}

Table 1 summarizes the characteristics of the sample. The majority of the participants were women $(66.4 \%)$. On average, the participants were $57.8(\mathrm{SD}=14.2)$ years of age and the disappearance took place $15.5(\mathrm{SD}=17.0)$ years earlier. Most of the participants experienced the disappearance of a child (30.6\%) and most participants $(32.8 \%)$ presumed that their relative disappeared due to a criminal act (e.g., kidnapping, homicide). More than half (61.2\%) of the participants assumed that their missing loved one was dead. The 134 participants represented 89 (66.4\%) unique missing persons.

\section{Measures}

\section{Dependent Variables}

The 19-item Inventory of Complicated Grief (ICG) was used to assess self-rated symptoms of PGD (Prigerson et al. 1995). Although the original 29-item ICG-r was 
Table 1 Characteristics of the participants $(n=134)$

\begin{tabular}{lr} 
Women, $N(\%)$ & $89(66.4)$ \\
Age (in years), $M(\mathrm{SD})$ & $57.8(14.2)$ \\
Educational level, $N(\%)$ & \\
Primary to moderate & $76(56.7)$ \\
High & $58(43.3)$ \\
Lost relative is, $N(\%)$ & \\
Partner/spouse & $18(13.4)$ \\
Child & $41(30.6)$ \\
Parent & $14(10.4)$ \\
Sibling & $31(23.1)$ \\
Other family member & $28(20.9)$ \\
Other & $2(1.5)$ \\
Number of years since disappearance, $M(\mathrm{SD})$ & $15.5(17.0)$ \\
Presumed cause of disappearance, $N(\%)$ & \\
Criminal act & $44(32.8)$ \\
Voluntarily & $33(24.6)$ \\
Accident & $33(24.6)$ \\
No specific suspicion & $24(17.9)$ \\
Belief about whereabouts of missing person, $N(\%)$ & \\
Alive & $25(18.6)$ \\
Dead & $82(61.2)$ \\
Doubt & $27(20.1)$ \\
Unique missing persons & $89(66.4)$ \\
\hline
\end{tabular}

administered (Boelen et al. 2003; Prigerson and Jacobs 2001), we only included the frequently used 19 items of the ICG (e.g., Hargrave et al. 2012) in order to prevent content overlap with items of other measures used within this study. Participants rated how frequently they experienced grief reactions during the last month, on a scale ranging from 0 ("never") to 4 ("always"). Total scores range from 0 to 76 . Items that referred to death were adapted to disappearance (e.g., "Ever since he/she has been missing it is hard for me to trust people"). The ICG has adequate psychometric properties (Prigerson et al. 1995). Cronbach's alpha in the current study was 0.92.

The 20-item PTSD Checklist for DSM-5 (PCL-5) was used to assess PTSD severity (Blevins et al. 2015; Boeschoten et al. 2014). The PCL-5 represents the DSM-5 PTSD criteria (APA 2013). Participants rated to what extent they experienced PTSD symptoms during the preceding month on a scale ranging from 0 ("not at all") to 4 ("extremely") (e.g., "How much were you bothered by feeling distant or cut off from other people?"). Total scores range from 0 to 80 . The wording "the stressful experience" in the instruction and the items was replaced by "the events associated with the disappearance". The psychometric properties of the PCL-5 are adequate (Blevins et al. 2015). Cronbach's alpha in the current study was 0.95 .

The 30-item Inventory of Depressive Symptomatology-Self-Report (IDS-SR) was used to assess MDD severity (Rush et al. 1996). Participants were instructed to choose 
one out of four options (range 0-3) indicating how frequently they experienced a symptom (e.g., "Feeling sad") during the past 7 days. A total score was obtained by summing up 28 out of the 30 items (range $0-84$ ). The IDS-SR showed good psychometric properties (Rush et al. 1996). Cronbach's alpha in the current study was 0.92 .

\section{Independent Variables}

The Grief Cognitions Questionnaire (GCQ) was used to assess negative cognitions related to the disappearance (Boelen and Lensvelt-Mulders 2005). Four of its nine subscales were assessed: self (six items; e.g., "I am ashamed of myself, since he/she has been missing", range 0-30), life (four items; "Life has got nothing to offer me anymore," range 0-20), future (five items; "My wishes for the future will never be fulfilled," range 0-25), and catastrophic misinterpretations of one's own grief reactions (four items; "If I let go of my emotions, I will go crazy," range 0-20). Participants rated their agreement with each item on 6-point scales (anchors: $0=$ disagree strongly and $5=$ agree strongly). Words that referred to death in the items were adapted to refer to the disappearance. The GCQ has adequate psychometric properties (Boelen and LensveltMulders 2005). Cronbach's alphas of the subscales were 0.90 (self), 0.93 (life), 0.92 (future), and 0.93 (catastrophic misinterpretations).

The Depressive and Anxious Avoidance in Prolonged Grief Questionnaire (DAAPGQ) was used to assess avoidance behaviors (Boelen and van den Bout 2010). The nine-item DAAPGQ consists of two subscales: depressive avoidance (five items; e.g., "I develop very few new activities since he/she has been missing, because I am unable to do so," range 0-25) and anxious avoidance (four items; e.g., "I avoid to dwell on painful thoughts and memories connected to his/her disappearance," range 020). Participants rated their agreement with each item on 6-point scale with anchors "not at all true for me" (0) to "completely true for me" (5). Words that referred to death in the items were adapted to refer to the disappearance. The DAAPGQ has adequate psychometric properties (Eisma et al. 2013). Cronbach's alpha was 0.92 for depressive avoidance and 0.75 for anxious avoidance.

\section{Sociodemographic Characteristics}

Gender, age, kinship (categorized as child/spouse versus other), number of years since disappearance, and educational level (categorized as primary to moderate versus high) were registered. The presumed cause of disappearance and belief about the whereabouts of the missing loved one were also assessed. Presumed cause of disappearance was categorized as follows: voluntary, victim of criminal act, victim of accident, and no (specific) suspicion. Belief about the whereabouts of the missing loved one was categorized as follows: I think (s)he is alive, I doubt whether (s)he is alive, and I think (s)he is not alive.

\section{Statistical Analyses}

Multilevel regression analyses were used to deal with the nested structure of the data. The nested structure of our data involves a two-level hierarchy: the participant (level 1, henceforth referred to as the participant level) is nested within a social network/family 
of other relatives of the same missing person (level 2, henceforth referred to as the family level). Intraclass coefficients were estimated to assess the proportion of variance at the family level, based on random intercept-only models with symptom levels (i.e., sum scores) of PGD, PTSD, or MDD as the respective dependent variable. In case no variance could be explained by the family level, single-level regression analyses were performed.

First, regression analyses were performed to examine whether each sociodemographic variable had a main effect on symptom levels of PGD, PTSD, and/or MDD. To this end, series of univariate analyses were performed with each of the sociodemographic variables as the independent variable, using dummy variables for the categorical variables, and symptom levels of PGD, PTSD, or MDD as the dependent variable.

Subsequently, the sociodemographic variables with a significant main effect on the indices of psychopathology were entered to the first model with symptom levels of PGD, PTSD, or MDD severity scores as dependent variable. The sum scores of the cognitive-behavioral variables were, next to the sociodemographic variables, entered to a second model. The explained proportion of variance at each level was computed for each model. A Bonferroni correction was used for the main analyses to correct for multiple tests, resulting in an alpha of $0.02(0.05 / 3)$.

\section{Results}

For PGD, 18.4\% of the variance was at the family level (level 2) and $81.6 \%$ at the participant level (level 1). For PTSD, $18.1 \%$ of the variance was at the family level and $81.9 \%$ at the participant level. For MDD, the variance at the family level was $0 \%$ and $100 \%$ at the participant level; thus, a single-level model was used in further analyses with respect to MDD.

\section{Fixed Main Effects of Sociodemographic Variables on Symptom Levels of PGD, PTSD, and MDD}

The results of the univariate regression analyses are displayed in Table 2. Women reported significantly higher levels of $\operatorname{MDD}(B=5.29, \mathrm{SE}=2.47, p=.034)$, but not PGD and PTSD, than men. Time since loss was significantly and inversely related to PGD $(B=-0.29, \mathrm{SE}=0.08, p<.001)$, PTSD $(B=-0.22, \mathrm{SE}=0.09, p=.019)$, and MDD $(B=-0.16, \mathrm{SE}=0.07, p=.019)$, indicating that psychopathology levels decrease with the passage of time. Also, type of kinship had a significant main effect on symptom levels of PGD, PTSD, and MDD. Those who experienced the disappearance of a child or spouse reported higher PGD $(B=11.24, \mathrm{SE}=2.35, p<.001)$, PTSD $(B=9.91, \mathrm{SE}=2.85, p=.001)$, and $\mathrm{MDD}(B=6.20, \mathrm{SE}=2.33, p=.009)$ levels compared with people whose parent, sibling, or more distant relative disappeared. Those who believed the missing loved one would be still alive reported significantly higher PGD $(B=8.97, \mathrm{SE}=3.42, p=.010)$ and PTSD $(B=8.23, \mathrm{SE}=4.03, p=.043)$ levels, not MDD levels, than those who believed that the missing loved one was deceased. Effects of age, educational level, and the type of disappearance (e.g., voluntarily missing versus presumed victim of a criminal act) were not statistically significant. 
Table 2 Fixed main effects of sociodemographic variables on PGD, PTSD, and MDD in relatives of missing persons $(n=134)$; each parameter results from a univariate regression

\begin{tabular}{|c|c|c|c|c|c|c|}
\hline \multirow[t]{2}{*}{ Variable } & \multicolumn{2}{|l|}{ PGD } & \multicolumn{2}{|l|}{ PTSD } & \multicolumn{2}{|l|}{ MDD } \\
\hline & $B$ & $\mathrm{SE}$ & $B$ & SE & $B$ & $\mathrm{SE}$ \\
\hline Gender & 1.83 & 2.62 & 4.62 & 3.04 & $5.29 *$ & 2.47 \\
\hline Educational level & -2.40 & 2.63 & -0.93 & 3.09 & 2.55 & 2.39 \\
\hline Time since loss (in years) & $-0.29 * * *$ & 0.08 & $-0.22 *$ & 0.09 & $-0.16^{*}$ & 0.07 \\
\hline Kinship & $-11.24 * * *$ & 2.35 & $-9.91 * *$ & 2.85 & $-6.20 * *$ & 2.33 \\
\hline \multicolumn{7}{|l|}{ Presumed reason of disappearance } \\
\hline Voluntarily versus criminal act & 5.94 & 3.53 & 3.85 & 4.19 & 1.65 & 3.18 \\
\hline Voluntarily versus accident & -3.88 & 3.84 & -4.19 & 4.55 & -1.61 & 3.39 \\
\hline Voluntarily versus no specific suspicion & 3.06 & 4.00 & -1.90 & 4.75 & 0.04 & 3.70 \\
\hline \multicolumn{7}{|l|}{ Whereabouts of missing loved one } \\
\hline Dead versus alive & $8.97 *$ & 3.42 & $8.23^{*}$ & 4.03 & 1.60 & 3.15 \\
\hline Dead versus doubt & 4.51 & 3.21 & 2.57 & 3.80 & 0.26 & 3.06 \\
\hline
\end{tabular}

Gender was coded as $0=$ men, $1=$ women; educational level as $0=$ primary to moderate, $1=$ other, kinship as $0=$ child or spouse, $1=$ other; "Voluntarily" was the reference category for "presumed reason of disappearance"; "Dead" was the reference category for "whereabouts of missing loved one"

$* p<.05 ; * * p<.01 ; * * * p<.001$

\section{Cognitive-Behavioral Correlates of PGD, PTSD, and MDD}

First, the sociodemographic variables that yielded significant main effects on symptom levels of PGD, PTSD, or MDD were entered simultaneously in the first model for PGD, PTSD, or MDD, respectively. In the second model, the cognitive-behavioral variables were added. Individual variance inflation factors were all below 5, suggesting no cause for concern about multicollinearity. The results are displayed in Table 3.

The sociodemographic variables explained $20.1 \%$ of the variance in PGD at the participant level. The type of kinship explained a unique proportion of the variance. The explained variance in PGD at the participant level increased to $64.3 \%$ by adding the cognitive-behavioral variables to the second model. Catastrophic misinterpretations of one's own grief reactions and depressive avoidance behavior were uniquely associated with PGD severity when taking into account the other variables.

The sociodemographic variables explained $11.9 \%$ of the variance in PTSD at the participant level. Type of kinship explained a unique proportion of the variance in PTSD. The explained variance in PTSD at the participant level increased to $70.9 \%$ by adding the cognitive-behavioral variables to the second model. Misinterpretations of one's own grief reactions and depressive avoidance were significantly associated with PTSD levels when taking into account the other variables. Because the PTSD measure included two PTSD avoidance symptoms, which may artificially inflate the strength of its relationships with indices of anxious avoidance and depressive avoidance, we repeated the multilevel analysis for PTSD, excluding the two PTSD avoidance symptoms (items 6 and 7). This analysis yielded similar results, indicating that misinterpretations of one's own grief reactions and depressive avoidance $(B=0.90, \mathrm{SE}=0.20$, 


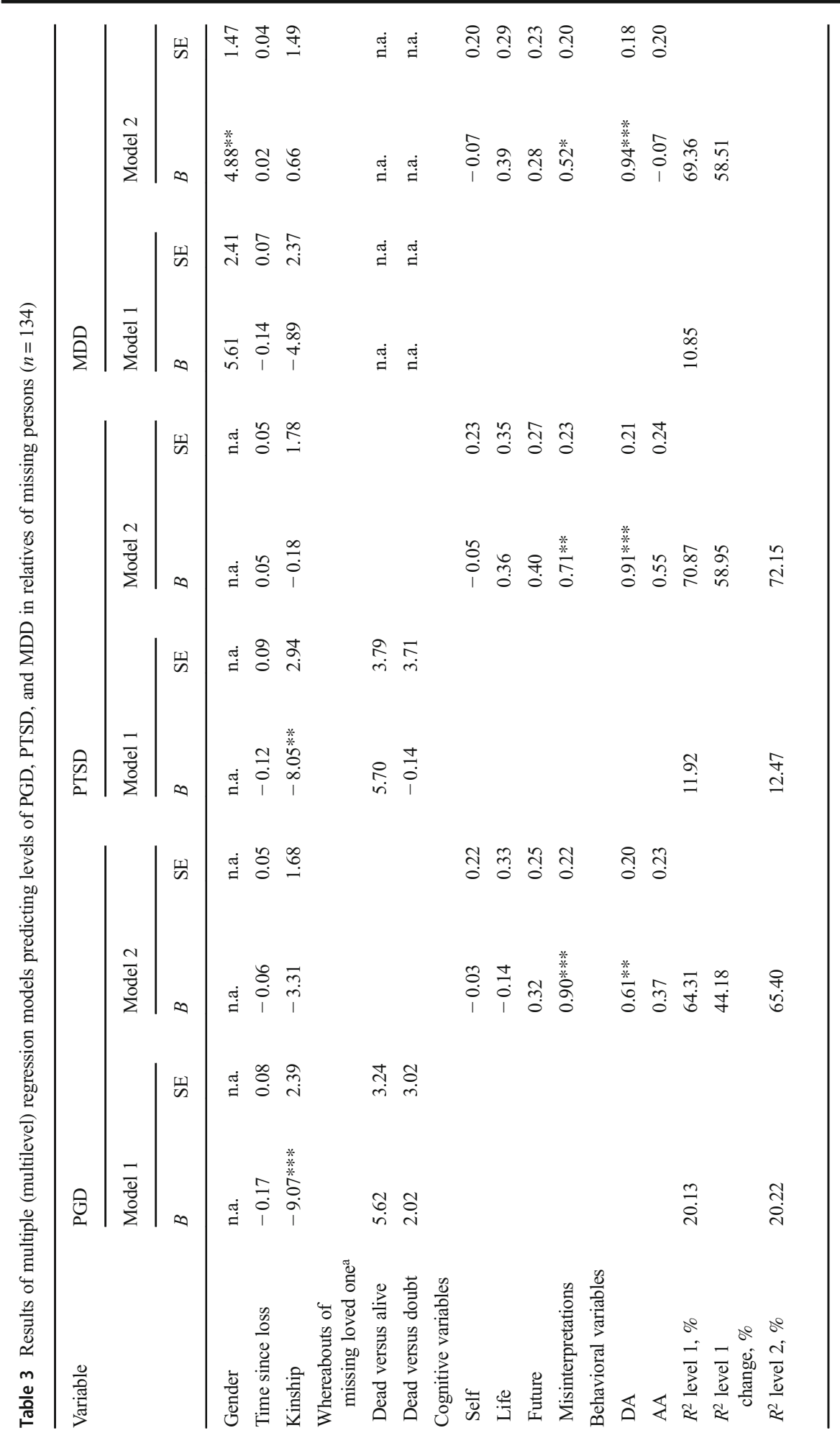




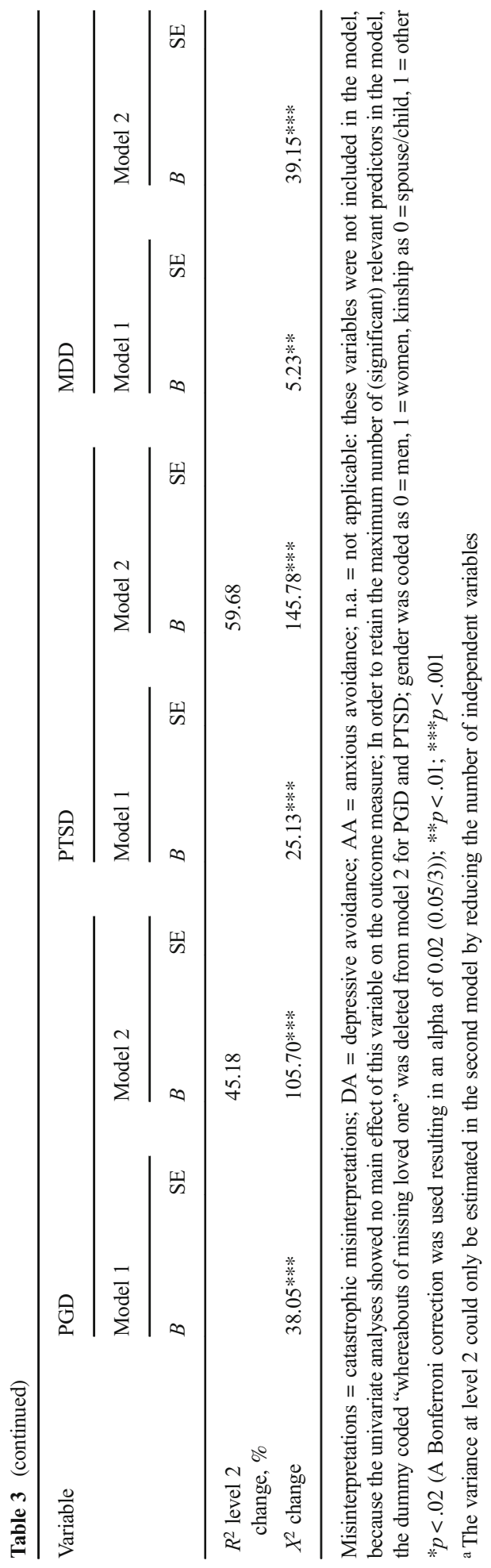


$p<.001)$, but not anxious avoidance $(B=0.41, \mathrm{SE}=0.22, p=.07)$, were significantly associated with PTSD levels when taking into account the other variables.

The sociodemographic variables explained $10.9 \%$ of the variance in MDD. The explained variance in MDD increased to $69.4 \%$ by adding the cognitive-behavioral variables to the model. Misinterpretations of one's own grief reactions and depressive avoidance behavior were uniquely associated with MDD levels when taking into account the other variables. Repeating the analysis with excluding three items (item 8,19 , and 21) of the depression measure that may overlap with the indices of anxious avoidance and depressive avoidance yielded similar results, indicating that depressive avoidance was significantly related to depression when taking into account the other variables $(B=0.77, \mathrm{SE}=0.16, p<.001)$.

\section{Discussion}

Our aim was to explore the generalizability of a cognitive behavioral model of disturbed grief (Boelen et al. 2006) for explaining PGD, PTSD, and MDD levels in relatives of long-term missing persons. Consistent with our hypothesis, the cognitivebehavioral variables explained a significant proportion of the variance in symptom levels of PGD, PTSD, and MDD, over and above relevant sociodemographic variables. Catastrophic misinterpretations of one's own grief reactions (but not negative cognitions about one's self, life, and future) and depressive avoidance behavior explained a unique proportion of the variance in PGD, PTSD, and MDD when taking the shared variance of other variables into account. Our findings are roughly in line with previous studies indicating that catastrophic misinterpretations of one's own grief reactions and depressive avoidance were most strongly associated with indices of psychopathology (Boelen et al. 2015; Boelen et al. 2016; Boelen and van den Bout 2010).

Taken together, the results indicate that relatives of missing persons, similar to people bereaved by the death of a loved one, experience elevated PGD, PTSD, and MDD levels when they engage in catastrophic misinterpretations of one's own grief reactions (e.g., "If I let go of my emotions, I will go crazy") and tend to withdraw from different social/recreational activities from the belief that these activities are pointless. These findings also suggest that relatives of missing persons, with elevated psychopathology symptoms, may benefit from addressing these negative cognitions and avoidance behavior in treatment. Cognitive behavioral therapy (CBT) may be the most obvious treatment option, since it has shown to be effective for treatment of postbereavement complaints (Currier et al. 2010). For example, CBT could enhance awareness of one's own misinterpretation of the grief reactions and could transform these maladaptive cognitions into more adaptive cognitions (e.g., by cognitive restructuring). Furthermore, CBT could promote relatives of missing persons to reengage in activities that were perceived as fulfilling prior to the disappearance (e.g., by behavioral reactivation). However, the effectiveness of a CBT-based intervention for relatives of missing persons has only been evaluated once (Hagl et al. 2014). Future research should further evaluate and optimize interventions for relatives of missing persons in need of professional support.

With respect to the sociodemographic correlates of CBT, our univariate analyses showed, among others, a main effect of belief about the whereabouts of the missing 
person. That is, participants who believed their missing loved one would be still alive reported significantly higher PGD and PTSD levels, not MDD levels, than those who believed that the missing loved one died. This finding is reminiscent of previous findings that the amount of hope that the missing loved one would be still alive was associated with increased PGD levels, but not with PTSD (C. Heeke, personal communication, October 31, 2017) and MDD levels (Heeke et al. 2015). However, a failure to reject the null hypothesis renders a finding inconclusive: the absence of a statistically significant effect may indicate that hope that a missing person is alive is less important for MDD levels or that our study was not able to detect the effect. Future research may shed more light on this issue.

More generally, it may be important to focus on the function of hope in treatment for relatives of missing persons (Lenferink et al. 2016). Although holding on to hope that the missing relative is alive may in some cases be more realistic (e.g., kidnapping cases or voluntarily missing persons), for those who maintain hope against all odds, holding on to hope may block acceptance of the irreversibility of the separation. Using exposure techniques in treatment might yield favorable effects, similar to effects of exposure for disturbed grief after bereavement (cf. Bryant et al. 2014).

It was salient that at the family level (level 2) zero variance in MDD was estimated, contrasting estimated variances at the family level in PGD and PTSD about $18 \%$. In other words, MDD symptoms may predominately be intrapersonal and thus independent from other relatives of the same missing person. A previous study among bereaved parents also showed a relatively low amount of variance in MDD explained by the family level compared with the variance levels in PGD (Wijngaards-de Meij et al. 2005). Clearly, this conclusion must remain tentative pending additional research, given that our sample represented a relatively small number of relatives of the same missing person.

Several further limitations need to be taken into account while interpreting the results of the current study. Self-report measures were used, which may lead to overestimation of symptom levels (Engelhard et al. 2007). Furthermore, the items of ICG are not identical to the proposed criteria of persistent complex bereavement disorder for the DSM-5 (APA 2013) or PGD criteria for the 11th edition of the International Classification of Diseases (Maercker et al. 2013); thus, the results of the current study may not directly speak to (future) studies using these criteria (Maciejewski et al. 2016). In addition, we used a convenience sample mostly recruited via (peer) support organizations. It may be that our sample constitutes individuals with more pervasive psychopathology levels compared with individuals who do not seek (peer) support (de Groot and Kollen 2013). Some caution is therefore warranted when generalizing the current findings to the population. Another limitation of the study sample is that the average time since disappearance was 15.5 years. Although time since disappearance was not strongly related to the outcome measures, we cannot rule out that our results may not be generalizable to people whose relative disappeared more recently. Lastly, it must be noted that the cross-sectional design precludes drawing conclusions about causality.

To conclude, this correlational study, focusing on cognitive-behavioral correlates of psychopathology post-disappearance for the first time, showed the generalizability of the cognitive-behavioral model of psychopathology post-loss to relatives missing persons. Our findings are consistent with other studies among bereaved samples, which 
suggest that it might be fruitful to evaluate the effectiveness of CBT for individuals who suffer from elevated psychopathology levels following the disappearance of a significant other.

Acknowledgements We would like to thank the "Vereniging Achterblijvers na Vermissing," "AVROTROS Vermist," Victim Support the Netherlands, and Child Focus for their support with the recruitment of relatives of missing persons. In addition, we would like to thank the Dutch police, especially Carlo Schippers and Irma Schijf, for their kind collaboration throughout this research project. Also, we give thanks to M. E. Timmerman for statistical consultation.

Authors' Contributions The first author LL designed the study, collected the data, performed the data analyses, and wrote the manuscript under supervision of JdK, IW, and PB. All authors contributed to and have approved the final manuscript.

Funding The Victim Fund, Foundation for the stimulation of bereavement research, and the University of Groningen supported this work.

Compliance with Ethical Standards Approval for conducting the study was obtained from a local ethics review board. All participants gave written informed consent.

Open Access This article is distributed under the terms of the Creative Commons Attribution 4.0 International License (http://creativecommons.org/licenses/by/4.0/), which permits unrestricted use, distribution, and reproduction in any medium, provided you give appropriate credit to the original author(s) and the source, provide a link to the Creative Commons license, and indicate if changes were made.

\section{References}

American Psychiatric Association. (2013). Diagnostic and statistical manual of mental disorders (5th ed.). Arlington: American Psychiatric Publishing.

Association of Chief Police Officers. (2010). Guidance on the management, recording and investigation of missing persons. London: Association of Chief Police Officers.

Beck, A. T. (1987). Cognitive models of depression. The Journal of Cognitive Psychotherapy: an International Quarterly, 1, 5-37.

Blevins, C. A., Weathers, F. W., Davis, M. T., Witte, T. K., \& Domino, J. L. (2015). The posttraumatic stress disorder checklist for DSM-5 (PCL-5): development and initial psychometric evaluation. J Trauma Stress, 28(6), 489-498.

Boelen, P. A., de Keijser, J., \& Smid, G. (2015). Cognitive-behavioral variables mediate the impact of violent loss on post-loss psychopathology. Psychol Trauma Theory Res Pract Policy, 7(4), 382-390.

Boelen, P. A., \& Eisma, M. C. (2015). Anxious and depressive avoidance behavior in post-loss psychopathology: a longitudinal study. Anxiety, Stress, \& Coping, 28(5), 587-600.

Boelen, P., \& Lensvelt-Mulders, G. (2005). Psychometric properties of the grief cognitions questionnaire (GCQ). J Psychopathol Behav Assess, 27(4), 291-303.

Boelen, P. A., \& van den Bout, J. (2010). Anxious and depressive avoidance and symptoms of prolonged grief, depression, and posttraumatic stress-disorder. Psychologica Belgica, 50(1/2), 49-68.

Boelen, P. A., van den Bout, J., de Keijser, J., \& Hoijtink, H. (2003). Reliability and validity of the Dutch version of the inventory of traumatic grief (ITG). Death Studies, 27(3), 227-247.

Boelen, P. A., van den Hout, M. A., \& van den Bout, J. (2006). A cognitive-behavioral conceptualization of complicated grief. Clin Psychol Sci Pract, 13(2), 109-128.

Boelen, P. A., van Denderen, M., \& de Keijser, J. (2016). Prolonged grief, posttraumatic stress, anger, and revenge phenomena following homicidal loss: the role of negative cognitions and avoidance behaviors. Homicide Studies, 20(2), 177-195.

Boeschoten, M. A., Bakker, A., Jongedijk, R. A., \& Olff, M. (2014). PTSS checklist voor de DSM-5 (PCL-5). Unpublished manuscript. 
Boss, P. (2006). Loss, trauma, and resilience: therapeutic work with ambiguous loss. New York: W.W. Norton \& Co..

Bryant, R. A., Kenny, L., Joscelyne, A., Rawson, N., Maccallum, F., Cahill, C., et al. (2014). Treating prolonged grief disorder: a randomized clinical trial. JAMA Psychiatry, 71(12), 1332-1339.

Currier, J. M., Holland, J. M., \& Neimeyer, R. A. (2010). Do CBT-based interventions alleviate distress following bereavement? A review of the current evidence. International Journal of Cognitive Therapy, 3(1), 77-93.

de Groot, M., \& Kollen, B. J. (2013). Course of bereavement over 8-10 years in first degree relatives and spouses of people who committed suicide: longitudinal community based cohort study. $\mathrm{Br} \mathrm{Med} J, 347,1-$ 11.

Ehlers, A., \& Clark, D. M. (2000). A cognitive model of posttraumatic stress disorder. Behav Res Ther, 38(4), 319-345.

Eisma, M. C., Stroebe, M. S., Schut, H. A. W., Boelen, P. A., van den Bout, J., \& Stroebe, W. (2013). Avoidance processes mediate the relationship between rumination and symptoms of complicated grief and depression following loss. J Abnorm Psychol, 122(4), 961-970.

Engelhard, I. M., van den Hout, M. A., Weerts, J., Arntz, A., Hox, J. J., \& McNally, R. J. (2007). Deploymentrelated stress and trauma in Dutch soldiers returning from Iraq. Prospective study. Br J Psychiatry, 191(2), $140-145$.

Hagl, M., Rosner, R., Butollo, W., \& Powell, S. (2014). Dialogical exposure with traumatically bereaved Bosnian women: findings from a controlled trial. Clinical Psychology and Psychotherapy, 22(6), 604 618.

Hargrave, P. A., Leathem, J. M., \& Long, N. R. (2012). Peritraumatic distress: its relationship to posttraumatic stress and complicated grief symptoms in sudden death survivors. J Trauma Stress, 25(3), 344-347.

Heeke, C., \& Knaevelsrud, C. (2015). Uneindeutiger verlust: Psychopathologische und psychosoziale konsequenzen im kontext gewaltsamer konflikte. Nervenarzt, 86(7), 826-832.

Heeke, C., Stammel, N., \& Knaevelsrud, C. (2015). When hope and grief intersect: rates and risks of prolonged grief disorder among bereaved individuals and relatives of disappeared persons in Colombia. $J$ Affect Disord, 173, 59-64.

Kristensen, P., Weisæth, L., \& Heir, T. (2012). Bereavement and mental health after sudden and violent losses: a review. Psychiatry, 75(1), 76-97.

Lenferink, L. I. M., de Keijser, J., Wessel, I., de Vries, D., \& Boelen, P. A. (2017). Toward a better understanding of psychological symptoms in people confronted with the disappearance of a loved one. Trauma, Violence, \& Abuse. https://doi.org/10.1177/1524838017699602.

Lenferink, L. I. M., Eisma, M. C., de Keijser, J., \& Boelen, P. A. (2017). Grief rumination mediates the association between self-compassion and psychopathology in relatives of missing persons. Eur $J$ Psychotraumatol, 8(sup6), 1378052.

Lenferink, L. I. M., van Denderen, M. Y., de Keijser, J., Wessel, I., \& Boelen, P. A. (2017). Prolonged grief and post-traumatic stress among relatives of missing persons and homicidally bereaved individuals: a comparative study. J Affect Disord, 209, 1-2.

Lenferink, L. I. M., Wessel, I., \& Boelen, P. A. (2018). Exploration of the associations between responses to affective states and psychopathology in two samples of people confronted with the loss of a loved one. $J$ Nerv Ment Dis, 206(2), 108-115.

Lenferink, L. I. M., Wessel, I., de Keijser, J., \& Boelen, P. A. (2016). Cognitive behavioural therapy for psychopathology in relatives of missing persons: study protocol for a pilot randomised controlled trial. Pilot and Feasibility Studies, 2(1).

Maciejewski, P. K., Maercker, A., Boelen, P. A., \& Prigerson, H. G. (2016). "Prolonged grief disorder" and "persistent complex bereavement disorder", but not "complicated grief", are one and the same diagnostic entity: an analysis of data from the Yale bereavement study. World Psychiatry, 15(3), 266-275.

Maercker, A., Brewin, C. R., Bryant, R. A., Cloitre, M., van Ommeren, M., Jones, L. M., et al. (2013). Diagnosis and classification of disorders specifically associated with stress: proposals for ICD-11. World Psychiatry, 12(3), 198-206.

Prigerson, H. G., Bierhals, A. J., Kasl, S. V., Reynolds, C. F., Shear, M. K., Newsom, J. T., \& Jacobs, S. (1996). Complicated grief as a disorder distinct from bereavement-related depression and anxiety: a replication study. Am J Psychiatr, 153(11), 1484-1486.

Prigerson, H. O., \& Jacobs, S. C. (2001). Traumatic grief as a distinct disorder: a rationale, consensus criteria, and a preliminary empirical test. In W. Stroebe, M. S. Stroebe, R. O. Hansson, \& H. Schut (Eds.), Handbook of bereavement research: consequences, coping, and care (pp. 613-645). Washington, D.C.: American Psychological Association. 
Prigerson, H., Maciejewski, P., Reynolds, C., Bierhals, A., Newsom, J., Fasiczka, A., et al. (1995). Inventory of complicated grief: a scale to measure maladaptive symptoms of loss. Psychiatry Res, 59(1), 65-79.

Rush, A. J., Gullion, C. M., Basco, M. R., Jarrett, R. B., \& Trivedi, M. H. (1996). The inventory of depressive symptomatology (IDS): psychometric properties. Psychol Med, 26(3), 477-486.

Shear, K., \& Shair, H. (2005). Attachment, loss, and complicated grief. Dev Psychobiol, 47(3), 253-267.

van der Houwen, K., Stroebe, M., Schut, H., Stroebe, W., \& van den Bout, J. (2010). Mediating processes in bereavement: the role of rumination, threatening grief interpretations, and deliberate grief avoidance. Soc Sci Med, 71(9), 1669-1676.

Wijngaards-de Meij, L., Stroebe, M., Schut, H., Stroebe, W., van den Bout, J., van der Heijden, P., \& Dijkstra, I. (2005). Couples at risk following the death of their child: predictors of grief versus depression. $J$ Consult Clin Psychol, 73(4), 617-623.

\section{Affiliations}

\section{Lonneke I. M. Lenferink ${ }^{1,2} \cdot$ Jos de Keijser $^{1} \cdot$ Ineke Wessel $^{1} \cdot$ Paul A. Boelen $^{2,3}$}

1 Department of Clinical Psychology and Experimental Psychopathology, Faculty of Behavioral and Social Sciences, University of Groningen, Grote Kruisstraat 2/1, 9712 TS Groningen, The Netherlands

2 Department of Clinical Psychology, Faculty of Social Sciences, Utrecht University, P.O. Box 80140, 3508 TC Utrecht, The Netherlands

3 Arq Psychotrauma Expert Group, Nienoord 5, 1112 XE Diemen, The Netherlands 